\title{
A Token Passing Tree MAC Scheme for Wireless Ad Hoc Networks to Support Real-Time Traffic
}

\author{
Rao Jianqiang ${ }^{1}$, Jiang Shengming ${ }^{2}$, and He Dajiang ${ }^{1}$ \\ 1 Department of Electrical Engineering, National University of Singapore, \\ 10 Kent Ridge Crescents, Singapore, 119260 \\ \{engp9040, engp8587\}@nus.edu.sg \\ 2 Centre for Wireless Communications, National University of Singapore, \\ 20 Science Park Road, \#02-34/37, Singapore, 117674 \\ jiangsm@cwc.nus.edu.sg
}

\begin{abstract}
This paper introduces a distributed MAC protocol which can provide delay-bounded service in wireless ad hoc networks. Ad hoc networks are network architectures that do not rely on a pre-existing fixed infrastructure, which imposes heavy challenges on designing MAC scheme with QoS support. Most existing MAC protocols for wireless ad hoc networks have been designed to support none-delay-sensitive applications. Given an increasing demand on supporting multimedia applications, there is much interest in designing MAC schemes to satisfy the requirements of real-time applications in ad hoc environments. The timed token based MAC scheme proposed in this paper allocates the bandwidth to the users according to their requirements. This scheme can guarantee the deadline of real-time traffic even in the case of heavy traffic load. In addition, a logical token-passing tree structure adopted in this scheme overcomes the hidden terminal problems in ad hoc networks.
\end{abstract}

\section{Introduction}

Since there is no need of preexisting infrastructure, a wireless ad hoc network can be rapidly deployed. Thus, such networks which can operate in different network conditions provide a low cost and flexible solution to communication networks. Most interest in this technology comes from both the commercial market and military applications. There are many challenges of the current research in this area. One of those issues is medium access control (MAC) which controls nodes to access medium and determines quality of service (QoS) and channel utilization [1]. Most existing MAC protocols which can provide QoS capability to users require some central entities, e.g., the base-stations in cellular wireless networks. Such schemes are not suitable in ad hoc environments because of the lack of central entities as mentioned above. Although a node can be selected to function as a central unit temporarily, this node cannot perform as robustly as a fixed one because of the possibility of platform movements. Therefore, the distributed MAC schemes are more desirable than the centralized MAC schemes used in cellular systems, although the latter support real-time traffic efficiently under the control of the central entity. 
Carrier sense multiple access (CSMA) is one of the most pervasive distributed MAC scheme used in wired network. But, Packet collisions are intrinsic to CSMA due to the nature of carrier sensing and the existence of hidden terminals in ad hoc networks. Many MAC schemes [2 3.4] either with or without carrier sensing, including the IEEE802.11 standard [5], have been proposed to improve the throughput over that of CSMA. These schemes handle collision avoidance by exchanging an in-band control packet (RTS/CTS) handshake prior to data transmission. The sender can transmit a data packet only after a successful RTS/CTS exchange. And collisions are solved by backing off and rescheduling RTS transmissions. These protocols, however, cannot meet the delay requirements of realtime traffic. The real-time packets may not be transmitted before their deadline because the control packets still suffer from collisions and their retransmissions are randomly scheduled.

Recently, several novel distributed MAC schemes have been proposed, aimed at providing the QoS guarantees for real-time traffic support. With the scheme proposed in [6], real-time nodes contend for access to channel with pulses of energy, the durations of which are function of the delay incurred by the nodes until the channel becomes idle. The resulting scheme guarantees priority to realtime traffic and provides round-robin service and bounded access delay to realtime nodes. Another scheme [7] uses window splitting protocols with limited feedback sensing to offer support for deadlines of real-time data. However, the two schemes mentioned above do not consider the existence of hidden terminals. The two other reservation-based schemes 89 can guarantee the contentionfree transmission based on the exchange of channel state informations among the active nodes. Of course, as the wireless spectrum is premium, the frequent exchange of network informations which may cause much overhead should be avoided.

In this paper, we introduce a Token Passing Tree (TPT) structure for indoor ad hoc networks, in which terminals have low mobility because of low movement speed (i.e., walking speed) and limited movement space. The possible scenarios include meeting rooms, wireless offices and other indoor environments where wiring is to be kept to minimum. A temporary ad hoc network can be rapidly constructed and used by users to exchange informations in such situation. The proposed MAC scheme is based on timed token protocol [10]. The timed token protocol is suitable for real-time communications not only because of its contention free nature but also due to the fact that it has the important property of bounded access time which is necessary for real-time communications. It is used pervasively in the wired network. However, the challenges on using the tokenpassing structure in the wireless ad hoc network comes from three aspects: the unreliable characteristics of wireless medium, the hidden terminals and the dynamics of nodes. The dynamics of nodes means that each node may switch off or on unpredictably. Addressing this, a Token Passing Tree structure is adopted, which is more easily maintained than traditional token passing scheme. Furthermore, a mechanism is provided to reconstruct the TPT when the token passing 
process is hampered due to the lost of the token. As long as a node maintains its position in a TPT, the QoS requirements of this node can be guaranteed.

In Section 2 we describe the MAC scheme and derive the condition to guarantee the bounded access delay to real-time node. The maintenance of TPT is also addressed in this section. Then, Section 3 presents the performance evaluation of proposed scheme. Finally, we conclude the paper in the last section.

\section{MAC Description}

The wireless ad hoc networks under consideration consist of $N$ nodes, approximately less than 30, all of which are functionally identical. Each node is trying to communicate with one another over a single hop, or with a access point or cluster head to reach a hidden nodes. In this case, each sender is within sight of the access point or cluster head. This is reasonable assumption for the hierarchical ad hoc networks, in which the network nodes are partitioned into clusters. As depicted in Figure 1 a typical TPT structure looks like a bi-directional tree with the token-rotation beginner (TRB) being the root. There are two logical tokenpassing directions: forward and backward. The former is used by a parent-node forwarding a token to its child-nodes while the latter is used by a child-node returning the token to its parent-node. The maintenance of TPT is presented in Section 2.2. Some parameters used in the following discussions are defined below.

- $T_{\text {prop }}$ is the propagation delay between a pair of nodes which is supposed to be identical to all nodes.

- $T_{\text {proc }}$ is the transmission time of a token frame.

- $H_{e}$ is the total reserved bandwidth of all nodes in TPT. $H_{e, i}$ is the bandwidth reserved by node $i$.

- $D$ is the common bounded access delay of nodes in TPT. $D_{i}$ is the bounded access delay of node $i$.

$-N$ is the total number of nodes in TPT.

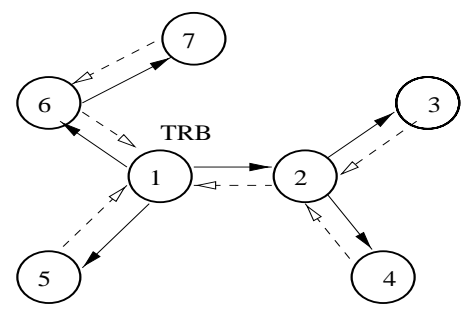

forward direction

$----\infty$

backward direction

Fig. 1. Typical structure of TPT 
A token frame contains the following information: a token sender ID (SID), which represents the identity of the node transmitting the packet; a token receiver ID (RID), which represents the identity of the node invited to transmit next; a Random Access Segment (RAS) flag, which is used in the procedure of node joining TPT; the configuration parameters of TPT $\left(D, N, H_{e}\right)$, which is used in TPT configuration. The token frame can be carried in the data packets to decrease the overhead caused by the token transmission. When a current token-holder transmits it last data packets, it add the ID of the node invited to transmit next in this packet. If the token-holder has no packet to send, a individual token frame is transmitted to the intended receiver in TPT. The ID of each node is given when it joins the TPT. After a node receives the token from its parent-node, it first detects whether the RAS flag is set. If the RAS flag is set, this node must wait a random access period $T_{\text {rap }}$ which is used by new nodes to submit request to join TPT. Then, it begins to transmit its data packet. The RAS flag is dynamically set to achieve the high channel utilization. In this scheme, we define that the RAS flag is set once every two cycles. And the nodes in TPT take their turns to set the RAS flag in the token frame.

\subsection{Bounded Access Delay with TPT}

Based on the timed token MAC protocol, a formed TPT can provide bounded access delay which is necessary to ensure that the transmission deadline of the real-time traffic is satisfied. The TPT adopts the mechanism used by the timed token protocol to allocate available bandwidth to the nodes. The timing properties of the timed token protocol shows that the average token rotation time is bounded by the Target Token Rotation Time (TTRT) and maximum token rotation time cannot exceed twice the TTRT. During the network configuration process, all nodes negotiate a common value for the bounded access delay $D$, an important parameter which guarantees the transmission deadline of the realtime traffic. Since each node has different bounded access delay requirements (denoted as $D_{i}$ for node $i$ ) to be satisfied, the negotiated bounded access delay $D$ should be the minimum value of all the $D_{i}$. that is,

$$
D=\min \left\{D_{i}, \forall i \in \Psi_{T P T}\right\}
$$

where $\Psi_{T P L}$ is the set of the nodes in a TPT.

Each node can reserve an amount of time (denoted as $H_{e, i}$ for node i) for transmission every time it receives a forward token. To guarantee bounded access delay $D$ to all nodes, based on the timed token protocol, the sum of $H_{e, i}$ allocated to each node can not exceed the Target Token Rotation Time, which equals the half of $D$. Therefore, the bandwidth allocation scheme can be expressed as follows,

$$
\sum_{\forall i \in \Psi_{T P L}} H_{e, i}+2(N-1)\left(T_{\text {proc }}+T_{\text {prop }}\right)+T_{\text {rap }} \leq \frac{D}{2}
$$

Once the node $i$ receives the forward token, the minimal time it can use is $H_{e, i}$. Then it can uses the left bandwidth only if the time elapsed since the 
previous token arrival is less than the value $\frac{D}{2}$. The amount of time (denoted as $H_{i}$ ) token holder $i$ can use is,

$$
H_{\max , i}=\left\{\begin{array}{c}
H_{e, i}+\frac{D}{2}-\left(T_{a, i}-T_{l a, i}\right) \\
H_{e, i}
\end{array} \begin{array}{c}
\left(T_{a, i}-T_{l a, i}\right) \leq \frac{D}{2} \\
\text { else }
\end{array}\right.
$$

where $T_{a, i}$ is the forward token arrival time of node $i, T_{l a, i}$ is the forward token last arrival time of the same node.

\subsection{TPT Configuration}
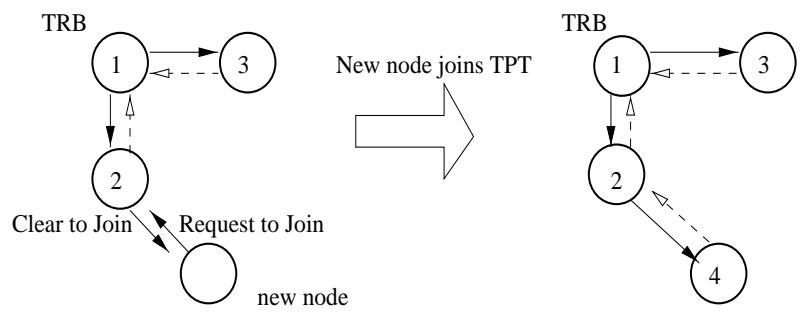

Fig. 2. Node joining procedure

Call Admission Control When a node becomes active, it first listens to the channel. If there is no TPT undergoing, this node can act as a TRB and construct a TPT with one node. Otherwise, it joins the current TPT as a new node. The new node can request to join the TPT only during the random access period (RAP). The duration of random access period $T_{\text {rap }}$ is longer than the sum of the length of a request to join (RTJ) and a clear to join (CTJ), plus a maximal round-trip propagation time. Figure 2 shows the procedure of a new node joining TPT. Suppose now, the node 2 in TPT returns the token to its parent-node 1 . The node 1 receives the token and waits $T_{\text {rap }}$ for new node joining TPT. Almost simultaneously, the new node can capture this token because the transmission of token is over radio channel, and get the address of node 2 from the token. Thereafter, it sends a RTJ to the node 2 during the RAP. The RTJ carries the bandwidth requirement of the new node $\left(D_{n e w}, H_{e, n e w}\right)$. The node 2 (landing node) receives the RTJ from the new node and performs the call admission control. This control determines whether the new call is admitted according to the requirement of new node, subject to the bounded access delay guaranteed by the current TPT. A new admission can occur if and only if the bounded access delay of the current TPT can be maintained. i.e,

$$
\overbrace{H_{e}+H_{\text {e,new }}}^{H_{\text {new }}}+2(\overbrace{N+1}^{N_{\text {new }}}-1)\left(T_{\text {proc }}+T_{\text {prop }}\right) \leq \overbrace{\frac{\overbrace{\min \left\{D, D_{\text {new }}\right\}}^{D_{\text {new }}}}{2} .} .
$$


Given that the condition is satisfied, the node 2 sends a clear to join (CTJ) to the new node and a unique ID is endowed with the new node. Then, the node 2 invokes the TPT configuration process (TPTCP) to inform the remaining node in TPT when it receives the token from the node 1 the next time. The token is set to be a configuration token which carries the new $D_{n e w}, N_{n e w}$ and $H_{e}$. The RAS flag in the token is disabled, which prevents other new nodes from sending the RTJ during the TPTCP. The TPTCP goes along with the normal token passing path. All other nodes in TPT can get the $D_{\text {new }}, N_{\text {new }}$ and $H_{e}$ from the configuration token. After the TPTCP token finishes its rotation, the node 2 forwards the token to the new node and the new nodes is added in the new TPT. The procedure of node joining TPT is parallel with the normal token passing operation which has no impact on the performance of this scheme except the overhead incurred by the RAP. It is possible that two or more new nodes sent the request to join the TPT simultaneously. Thus, the RTJs sent by these nodes will collide. To resolve the collisions, the RTJs are randomly scheduled to be retransmitted if the new nodes do not receive the CTJs from the landing node in TPT.

\subsection{Token Loss}

Communication failures in the wireless environment are a relatively frequent occurrence. With the proposed MAC scheme, the communication failures may be caused by the token passing failures, due to transmission error and/or node's powering off. For the token passing structure to be feasible in such environments, the TPL should be recovered without causing much overhead. In the case of a fixed network coordinator, the polling scheme is adopted. While in a distributed system each node needs to achieve a common decision on who should be responsible to recover the TPL in the case of token passing failure. For this reason, this scheme adopts a timer to detect the token passing failures and ensure that the TPL is recovered. This timer is set by each node in a distributed manner. The allocation scheme given in Section 2.1 can guarantee the interval between two successive token's arrivals at a node shorter than the common bounded access $D$. Thus a node sets a timer $T_{\text {timer }}$ equal to $D$ each time it receives the token. Then, $T_{\text {timer }}$ counts down until the token arrives at this node next time. If the token arrives at this node before its timer expires, $T_{\text {timer }}$ is reinitialized to $D$ and enabled again(starting the counting down process). If the token does not arrive before its timer expires (i.e., $T_{\text {timer }}=0$ ), it means that the token passing failure happens. This node regenerates a token and continues the token token passing loop. If it receives the token in the following loop, the TPT is recovered. Otherwise, the link between this node and its parent node is considered being broken. Then, it becomes a TRB and broadcasts this message to the other nodes. The broadcasted message can reach all hidden nodes of this node with the help of cluster or the access point. Then, the original TPT scheme is switched to the contention based MAC scheme, each node can join the TPT as a new node using the exchange of RTJ/CTJ with the nodes in new TPT. Because each node has 
its unique timer, only the node which first detects the token lost is chosen as TRB.

Considering the case of a network without the cluster, the broadcasted message may not reach all nodes in TPT before their timer expire. Thus, it is possible that two or more nodes become the TRB because their timer expire. Then, several TPTs may be constructed by these TRBs. We call this issue multi-TPT. The multi-TPT also exits in the multi-hop ad hoc networks. With the multi-TPT, the better space reuse can be achieved. But, at the same, it exits the problem that the token rotation of one TPT may interfere with the token rotation of its neighbor's TPT. Therefor, the maintenance of the multi-TPT is a much more complex open problem and is my undergoing work.

\section{Performance Evaluation}

The channel capacity of channel in wireless ad-hoe network is less than $10 \mathrm{Mb} / \mathrm{s}$, while the timed token protocol is generally adopted in the high bandwidth wired network $(100 \mathrm{Mb} / \mathrm{s})$. Thus, we have simulated this scheme over a variety of network configurations. The overall performance of this scheme in the steady state with a mixed population of data and real-time nodes is studied with simulations. We mainly investigate the effect of the two parameters, the bounded access delay of real time traffic and the data packet size. There are 10 real-time nodes, 20 data nodes in our simulation model. Table 1 shows the traffic model of real-time and data nodes. The channel bit rate $r_{c}$ of system is $2 \mathrm{Mb} / \mathrm{s}$. The traffic arrival rate $r_{s}$ of each real-time node is $64 \mathrm{~kb} / \mathrm{s}$. We assume that real-time packets are presented to the MAC layer periodically. Thus, the assembled packet size $\left(b_{r p k t}\right)$ is the product of the inter arrival rate $\left(A_{r}\right)$ of the real-time packet and the source rate $r_{s}$. The bounded access delay $D$ equals the deadline of real-time packet, which equals $A_{r}$. The reserved bandwidth of each real-time node equals the transmission time of a real-time packet. We can calculate that the total reserved bandwidth of real-time nodes is less than $\frac{D}{2}$. The other parameters are shown as follows. The size of token frame is 20 bytes. The $T_{\text {rap }}$ is $0.1 \mathrm{~ms}$. The propagation delay $T_{\text {prop }}$ is $0.1 u \mathrm{~s}$. The data load is defined as $N_{\text {data }} \lambda b_{p k t} / r_{c}$, where $N_{\text {data }}$ is the number of data station. Because the packet size is fixed, the delay is defined as the access delay. The throughput of data traffic is measured as the normalized data packets transmitted over the channel.

Because the real-time nodes are guaranteed with bounded access delay $D$ in the steady state as shown in previous section, it shows that the maximal realtime packet delay is less than the deadline of packet. No real-time packets are dropped even in high data load. Figure 3 shows the average real-time packet delay against the data load. The assembled real-time packet size is 1600bit, 3200bit and 6400 bit respectively. Accordingly, the bounded access delay $D$ is $25 \mathrm{~ms}, 50 \mathrm{~ms}$ and $100 \mathrm{~ms}$. The result shows that the average real-time packet delay is less than one third of the deadline of the packet even in the high data load. Given the same data load, the delay of real-time packet increases as its size increases. Figure 4 shows the throughput of data traffic against the data load. The contention free 


\begin{tabular}{|l||l|l|}
\hline Parameters & Real-time node & Data node \\
\hline \hline Interarrvial pdf & Constant & Poisson \\
\hline Interarrvial rates $(\mathrm{ms})$ & $A_{r}$ & $\lambda$ \\
\hline Packet length pdf & Constant & Constant \\
\hline Mean packet size(bytes) & $b_{r p k t}$ & $b_{p k t}$ \\
\hline Reserved bandwidth & $b_{r p k t} / r_{c}$ & 0 \\
\hline Packet deadline(ms) & $D$ & no requirement \\
\hline
\end{tabular}

Table 1. Traffic models

nature of token passing scheme ensures that every transmission is successful in a distortion free channel. The throughput can therefor reach a maximal value and is not degraded under heavy loads. We can see from Figure 3 and 4 that there is a tradeoff between the QoS requirements of real-time traffic and the throughput of the data traffic. The throughput of data traffic decreases as the bounded access delay $D$ decreases when the network is over loaded, while the smaller value of the bounded access delay means better QoS that the real-time traffic can get. The reason is that the transmission of data packet is constrained by the bounded access delay [11. Similarly, Figure 5 shows the impact of guaranteeing priority to real-time traffic on the average delay of data packet. The average data packet delay increases more quickly with the larger $D$ as the data load increases.

Figure 6] and 7] compares the average data packet delay and throughput of data traffic between the cases of different fixed-length data packet. We observe that the performance of this scheme is better when the data packet are shorter. The shorter data packet is more desirable to achieve better performance.

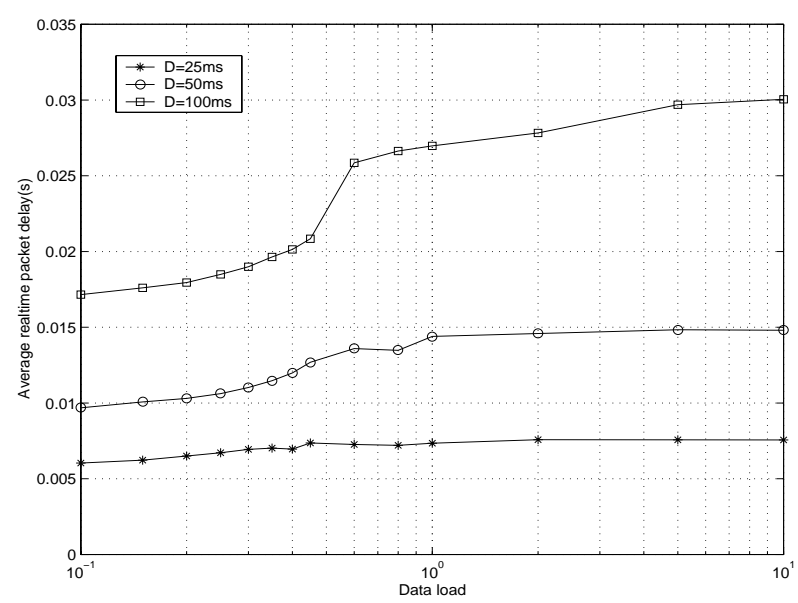

Fig. 3. Real-time packet delay versus data load 


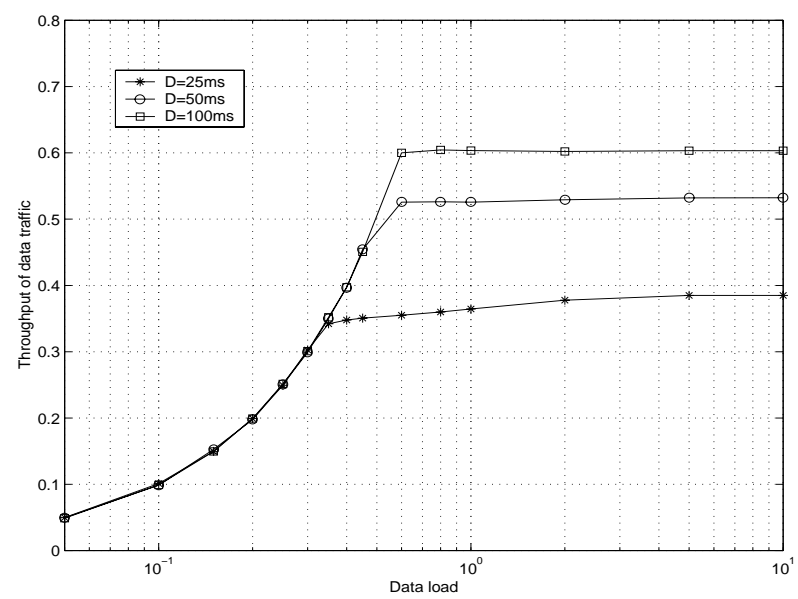

Fig. 4. Throughput of data traffic versus data load

\section{Conclusions}

We have described the structure of the token passing based MAC scheme for ad hoc networks, and investigated its major performance through simulation. The result have shown that this scheme can effectively support real-time traffic in the steady state. The ongoing work is to investigate the impact of the token lost events on this scheme and do the detailed performance analysis of this model. Our future studies will focus on improving the adaptivity of current MAC scheme.

\section{References}

1. M. Conti, C. Demaria and L. Donatiello.: Design and Performance Evaluation of A MAC Protocol for Wireless Local Area Networks. ACM Mobile Networks and Application, Vol. 2, No. 1, 1997, pp69-87.

2. P.Karn, MACA-A new channel access method for packet radio.: Proc. ARRL/CRRL Amateur Radio Ninth Computer Networking Conf, 1990.

3. F. Talucci and M. Gerla.: MACA-BI (MACA By Invitation) A Wireless MAC Protocol for High Speed Ad hoc Networking. The 6th IEEE International Conference on Universal Personal Communications, 1997, pp913-917.

4. C.L. Fullmer and J.J. Garcia-Luna-Aceves.: Floor Acquisition Multiple Access (FRMA) for Packet-Radio Networks. Proceedings of ACM SIGCOMM, 1994.

5. IEEE 802.11. Draft Standard for Wileless LAN. P802.11D5.0, 1996

6. J.L.Sobrinho and A.S.Krishnakumar.: Real-Time Traffic over the IEEE 802.11 Medium Access Control Layer. Bell Labs Technical Journal, Vol: 1, No.2, Autumn 1996, pp172-187. 


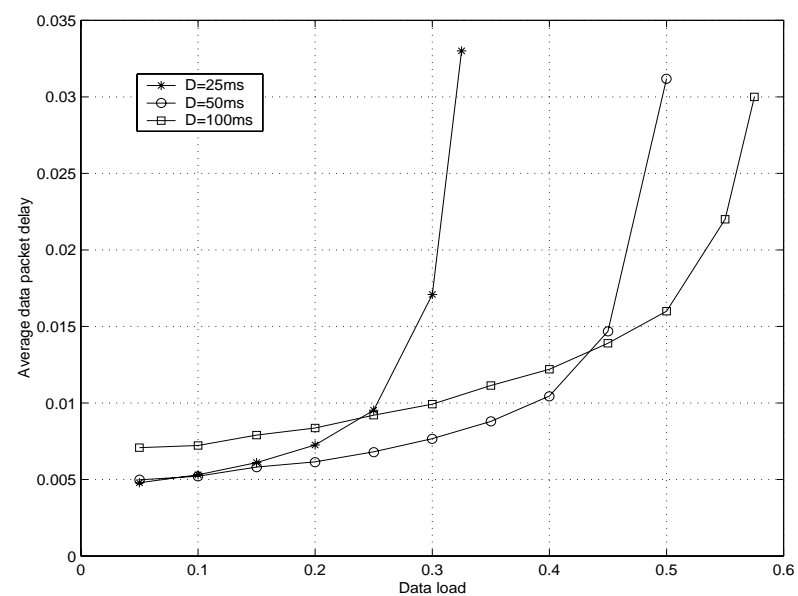

Fig. 5. Data packet delay versus data load

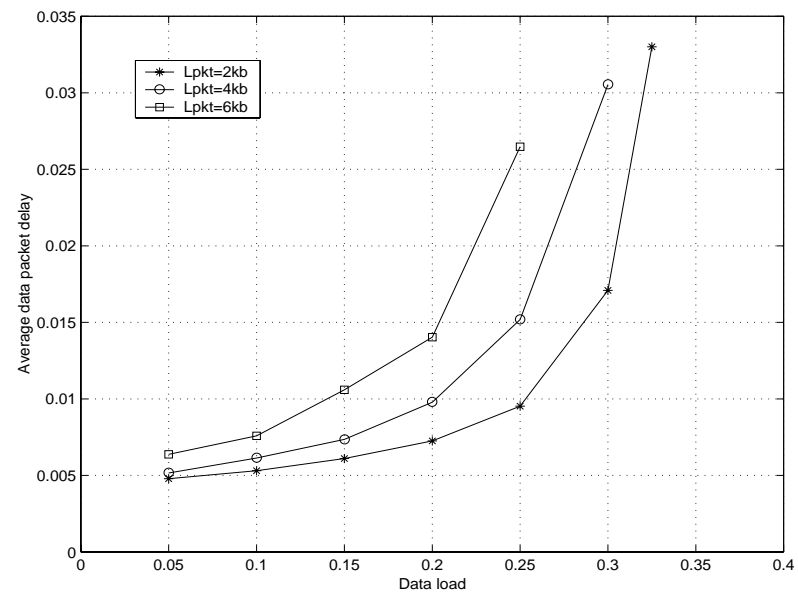

Fig. 6. Data packet delay versus packet length 


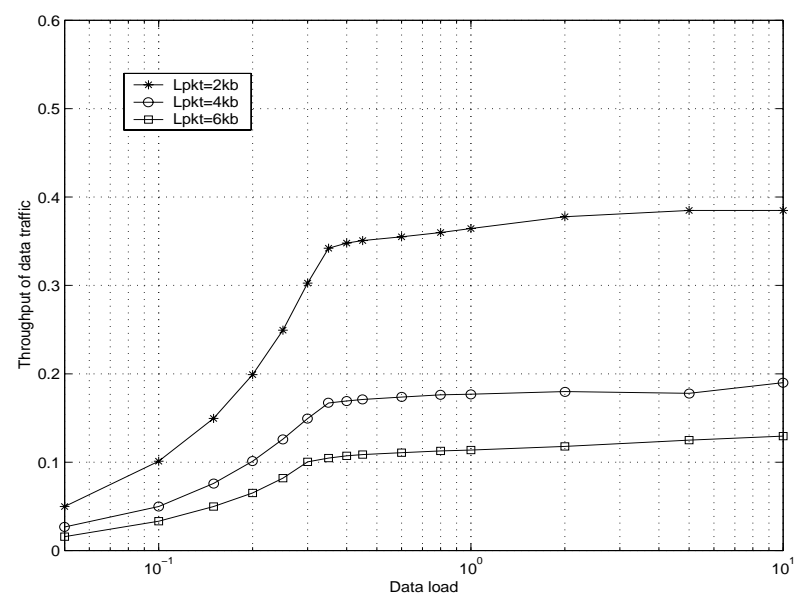

Fig. 7. Throughput of data traffic versus the data load

7. Michael J.Markowsk and Adarshpal S.Sethi.: Fully Distributed Wireless Transmission of Heterogeneous Real-Time Data. Vehicular Technology Conference, Volume: 2, 1998, pp1439-1442.

8. C.R. Lin and M. Gerla.: Asynchronous Multimedia Multimedia Radio Network. IEEE INFOCOM'97, 7-11 March, 1997.

9. A.Muir and J.Garcia-Luna-Aceves.: Supporting real-time multimedia traffic in a wireless LAN. Proc. SPIE multimedia Computing and Networking, pp.41-45 1997.

10. R.M.Grow.: A timed token protocol for local area networks. Proc.Electro/82, Token Access Protocols, May 1982.

11. Seung Ho Hong.: Approximate analysis of timer-controlled priority sceme in the single-service token-passing system. Networking,IEEE/ACM Transaction, Volume:22, April 1994, pp.206-215. 